\title{
FRECUENCIA DE SERORREACTORES A Lawsonia intracellularis EN GRANJAS PORCINAS TECNIFICADAS
}

\author{
Frequency of Seroreactors to LaWsonia intracellularis in Commercial \\ Pig FARMS
}

\author{
Astrid Calderón A. ${ }^{1,2}$, Sonia Calle E. ${ }^{1,3}$, Marlon Torres A. ${ }^{4}$, Siever Morales C. ${ }^{1}$ \\ y Chris Pinto $\mathbf{J}^{1}$
}

\section{RESUMEN}

El objetivo del estudio fue detectar la presencia de anticuerpos contra Lawsonia intracellularis, en granjas tecnificadas de los departamentos de Ica, Arequipa, La Libertad y Lambayeque. Los anticuerpos fueron detectados mediante la prueba de Inmunofluorescencia Indirecta (IFI). Se evaluaron 293 animales en varias etapas de producción. El $5.5 \pm 0.6 \%$ de los sueros analizados fueron positivos a L. intracellularis, habiendo el mayor porcentaje de serorreactores en el departamento de La Libertad (14.6\%), y la etapa productiva más afectada fue la de hembras reproductoras $(23.1 \%)$. Los resultados demuestran que la $L$. intracellularis es un agente etiológico de enteritis que afecta a la industria porcina en el Perú.

Palabras clave: Lawsonia intracellularis, enteropatía proliferativa porcina, inmunofluorescencia indirecta

\section{ABSTRACT}

The objective of this study was to detect the presence of Lawsonia intracellularis antibodies in commercial swine herds in the Peruvian departments of Ica, Arequipa, La Libertad and Lambayeque. The antibodies were detected using the Indirect Immunofluorescence Antibody Technique (IFAT) in serum samples of 293 animals in five different age groups. La Libertad department was the most affected (14.6\%) and female breeders were the most affected age group (23.1\%). Positive results were found in $5.5 \pm$ $0.6 \%$ of the samples. The results showed that $L$. intracellularis is an enteric ethiologic agent that affects the commercial swine production system of Peru.

Keywords: Lawsonia intracellularis, proliferative enteropathy, pigs, indirect inmunofluorescence

\footnotetext{
1 Laboratorio de Microbiología y Parasitología Veterinaria, ${ }^{4}$ Laboratorio de Producción Agropecuaria, Facultad de Medicina Veterinaria, Universidad Nacional Mayor de San Marcos, Lima

2 E-mail: astridvet@gmail.com

3 E-mail: calleson@gmail.com
} 


\section{INTRODUCCIÓN}

La Enteropatía Proliferativa Porcina (EPP) es una enfermedad de distribución mundial causada por una bacteria intracelular, actualmente denominada Lawsonia intracellularis. Esta bacteria se transmite vía fecal-oral, localizándose en el interior de los enterocitos de la cripta ileal. Se replica y se transmite fácilmente, de animal a animal y por fomites, aves o roedores, causando diarreas persistentes, desuniformidad en los pesos y un menor rendimiento productivo en las etapas de engorde, acabado, hembras de reemplazo y reproductoras. Esto se traduce en un impacto económico negativo en la industria porcina, debido al consumo extra de alimento, el retardo en los días de salida al mercado de los animales afectados y los gastos en tratamiento (McOrist, 1996; Lawson y Gibhart, 2000).

Esta enfermedad afecta a los porcinos, observándose una mayor frecuencia en granjas tecnificadas y en animales de líneas híbridas, aumentando el riesgo en situaciones de estrés (Machuca et al., 2002). Se ha reportado en una gran cantidad de países, tales como Australia, Bélgica, Canadá, Inglaterra, Estados Unidos, Dinamarca, México, Venezuela, Brasil, Argentina (Chiriboga et al., 1999). Un estudio en Lima, Perú, reveló un 39\% de seroprevalencia, lo cual denota una presencia importante de la enfermedad por lo que se requiere evaluar su situación en otras regiones del país (Valdez, 2001). El objetivo del presente estudio fue evaluar la frecuencia de animales serorreactores a Lawsonia intracellularis en granjas porcinas tecnificadas de zonas productoras de porcinos.

\section{Materiales y Métodos}

Para determinar el tamaño de muestra se utilizó el Teorema de Límite Central, resultando como mínimo un número de 30 animales por granja en seis granjas porcinas tecnificadas de los departamentos de Ica, Arequipa, La Libertad y Lambayeque. En la práctica, se colectó 293 muestras, las cuales fueron distribuidos en cinco grupos según las etapas de producción: gorrinos en recría, en crecimiento, en acabado, hembras reproductoras de diversos partos y hembras de reemplazo.

Se tomaron muestras de sangre $(5 \mathrm{ml})$, mediante punción de la vena cava anterior, y se trasladaron para su procesamiento al Laboratorio de Microbiología de la Facultad de Medicina Veterinaria, Universidad Nacional Mayor de San Marcos, Lima. Los sueros se analizaron por el método de Inmunofluorescencia Indirecta (IFI), utilizando el Kit comercial "Ileitest" y vistos a través del microscopio de fluorescencia (100x).

Se evaluó la frecuencia de serorreactores a $L$. intracellularis según zona, edad productiva y procedencia. La asociación de las variables en estudio y la frecuencia de serorreactores a la bacteria se analizaron mediante la prueba de Chi Cuadrado.

\section{Resultados}

En el $5.5 \pm 0.6 \%(16 / 293)$ de sueros se encontró la presencia de anticuerpos contra L. intracellularis. La mayor frecuencia de animales serorreactores se encontró en el departamento de La Libertad (14.6 $\pm 2.9 \%)$ (Cuadro 1). Así mismo, el mayor porcentaje de animales seropositivos fueron del grupo de reproductoras $(23.1 \pm 6.1 \%)$. Se encontró una asociación significativa entre la frecuencia de serorreactores con la procedencia y la etapa productiva $(\mathrm{p}<0.05)$ (Cuadro 2$)$.

\section{Discusión}

La presencia de anticuerpos a Lawsonia intracellularis en las diversas etapas productivas indican una anterior exposición de los animales a la bacteria. El presen- 
Cuadro 1. Frecuencia de cerdos con anticuerpos contra Lawsonia intracellularis en cuatro departamentos del Perú

\begin{tabular}{lccc}
\hline \multirow{2}{*}{ Departamento } & \multicolumn{2}{c}{ Animales } & $\begin{array}{c}\text { Frecuencia } \\
\left(\% \pm \text { I.C. }^{1}\right)\end{array}$ \\
\cline { 2 - 3 } & Muestreados (n) & Positivos (n) & $1.5 \pm 0.6$ \\
Ica & 134 & 2 & $14.6 \pm 2.9$ \\
La Libertad & 89 & 13 & 0 \\
Arequipa & 37 & 0 & $3 \pm 0.8$ \\
Lambayeque & 33 & 1 & $5.5 \pm 0.6$ \\
\hline Total & 293 & 16 & \\
\hline
\end{tabular}

${ }^{1}$ Intervalo de confianza del 95\%

Cuadro 2. Frecuencia de cerdos con anticuerpos contra Lawsonia intracellularis, según su etapa productiva en cuatro departamentos del Perú

\begin{tabular}{lccc}
\hline \multirow{2}{*}{ Etapa } & \multicolumn{2}{c}{ Animales } & $\begin{array}{c}\text { Frecuencia } \\
\left(\% \pm \pm \text { I.C. }{ }^{1}\right)\end{array}$ \\
\cline { 2 - 3 } & Muestreados (n) & Positivos (n) & 0 \\
Recría & 40 & 0 & 0 \\
Crecimiento & 109 & 0 & $3.3 \pm 0.7$ \\
Acabado & 59 & 2 & $6.1 \pm 1.9$ \\
Reemplazo & 33 & 2 & $23.1 \pm 6.1$ \\
Reproductoras & 52 & 12 & $5.5 \pm 0.6$ \\
\hline Total & 293 & 16 & \\
\hline
\end{tabular}

${ }^{1}$ Intervalo de confianza del 95\%

te estudio determinó su frecuencia en cuatro departamentos del Perú, siendo el primer estudio donde se ha logrado demostrar la presencia de serorreactores en granjas tecnificadas del norte del Perú.

El $5.5 \%$ de frecuencia encontrada fue bastante más bajo de otros reportes en granjas tecnificadas en la zona de Lima (39\%; Valdez, 2001). Asimismo, estudios de seroprevalencia en otros países muestran ín- dices más elevados: 22\% en España (Lanza y Pozo, 1996), 22\% en Brasil (Chiriboga et al., 1999; Ristow et al., 2001), 23\% en Venezuela (Kwiecien et al., 2001) y 30\% en Argentina (Machuca et al., 2002).

El bajo nivel de anticuerpos contra esta bacteria se debe a su ubicación en el interior de enterocitos inmaduros, hecho que conlleva a inconvenientes para el diagnóstico serológico (Lawson et al., 1988). El IFI es 
una prueba que detecta niveles de IgG desarrollados por el animal cuando está expuesto a L. intracellularis (Knittel et al., 1998); sin embargo, hay que considerar que esta prueba detecta seropositivos luego de dos semanas de haber ocurrido la infección, de modo que se obtiene resultados negativos en muestras colectadas con anterioridad.

Se encontró animales seropositivos únicamente en la etapa de acabado $(3.3 \pm 0.7 \%)$. Se conoce que la enfermedad ocurre típicamente en gorrinos de crecimiento y acabado luego de la caída de la inmunidad materna (Guedes et al., 2001); sin embargo, la ausencia de animales seropositivos en la etapa de crecimiento en el presente estudio podría deberse al mayor empleo de antibióticos en los últimos años para el control de esta bacteria (Schwartz, 2004). Estos antibióticos no se utilizan en la fase de acabado por los costos que demandan.

E1 $23 \pm 6.1 \%$ de seropositivas encontrado en las reproductoras indica que las madres son portadoras y se convierten en una importante fuente de infección. El incremento de la edad favorece la presencia de un mayor número de animales con anticuerpos contra L. intracellularis (Bronsvoort et al., 2001).

\section{Conclusiones}

Se detectó una frecuencia de $5.5 \pm 0.6 \%$ de serorreactores positivos a la bacteria Lawsonia intracellularis en los departamentos de Ica, Arequipa, La Libertad y Lambayeque.

\section{Literatura Citada}

1. Chiriboga A, Guimaraes W, Vanetti M, Araujo E. 1999. Detection of Lawsonia intracellularis in faeces of swine on the main producing regions in Brazil. Can $\mathrm{J}$ Microbiol 45: 230-234.
2. Guedes R, Gebhart C. 2001. Ileitis: Evaluation and interpretation of serological results. Proc Allen D Leman Swine Conference. Iowa: American Association of Swine Veterinarians. $p$ 111-114.

3. Knittel J, Jordan D, Schwartz K, Janke B, Roof M, McOrist S, Harris D. 1998. Evaluation of antemortem polymerase chain reaction and serological methods for detection of Lawsonia intracellularis exposed pigs. Am J Vet Res 58: 722-726.

4. Kwiecien E, Bermudez U, McOrist S. 2001. Detection of Lawsonia intracellularis in Venezuela pig farms. Proc Allen D Leman Swine Conference. Iowa. American Association of Swine Veterinarians. p 247-249.

5. Lanza I, Pozo J. 1996. Epidemiología de la ileitis porcina. Porci Vet 32: 42-49.

6. Lawson G, Gebhart C. 2000. Proliferative enteropathy. J Comp Path 122: 77-100.

7. Machuca M, Riganti J, Perfumo C. 2002. Actualización sobre una enfermedad emergente de los cerdos: La enteropatía proliferativa. Rev Med Vet 87: 53-58.

8. Machuca M, Riganti J, Puerta J, Venturini C, Sanguinetti H, Risso M, Perfumo C. 2002. A survey of Lawsonia intracellularis antibodies in fattener pigs in Argentina. Proc 17 $7^{\text {th }}$ IPVS Congress. Ames-Iowa. 2: 194.

9. McOrist S. 1996. Treatment and prevention of porcine proliferative enteropathy with oral tiamulin. Vet Rec 139: 615-618.

10. Ristow L, Silva L, Pérez A. 2001. Levantamento serologico da enteritis proliferativa dos suinos (ILEITE) no estado de Minas Gerais. X Congreso Brasilero de Veterinarios Especialistas em Suínos. Goiania.

11. Schwartz K. 2004. Current status of "Ileitis" in the midwest. Proc Swine Disease Conference for Swine Practicioners. Iowa. p 25-34. 
1. Valdez M. 2001. Detección de anticuerpos contra Lawsonia intracellularis en porcinos provenientes de granjas tecnificadas del valle de Lima y
Huaral. Tesis de Médico Veterinario. Lima: Facultad de Medicina Veterinaria, Univ. Nacional Mayor de San Marcos. Lima. $36 \mathrm{p}$. 